\title{
A Non-Perturbative Operator Product Expansion
}

\author{
W. Bietenholz*a , N. Cundy ${ }^{\mathrm{b}}$, M. Göckeler ${ }^{\mathrm{b}}$, R. Horsley ${ }^{\mathrm{c}}$, \\ H. Perlt ${ }^{\mathrm{d}}$, D. Pleiter ${ }^{\mathrm{e}}$, P.E.L. Rakow ${ }^{\mathrm{f}}$, G. Schierholz ${ }^{\mathrm{b}, \mathrm{g}}$, \\ A. Schiller ${ }^{d}$, T. Streuer $^{b}$ and J.M. Zanotti ${ }^{c}{ }^{\dagger}$ \\ a Insituto de Ciencias Nucleares, Universidad Nacional Autónoma de México \\ A.P. 70-543, C.P. 04510 Distrito Federal, México \\ ${ }^{\mathrm{b}}$ Institut für Theoretische Physik, Universität Regensburg, 93040 Regensburg, Germany \\ c School of Physics, University of Edinburgh, Edinburgh EH9 3JZ, United Kingdom \\ ${ }^{\mathrm{d}}$ Institut für Theoretische Physik, Universität Leipzig, 04109 Leipzig, Germany \\ e John von Neumann Institut für Computing NIC, \\ Deutsches Elektron-Synchrotron DESY, 15738 Zeuthen, Germany \\ f Theoretical Physics Division, Dept. of Mathematical Sciences, University of Liverpool, \\ Liverpool, L69 3BX, United Kingdom \\ g Deutsches Elektron-Synchrotron DESY, 22603 Hamburg, Germany \\ E-mail: wolbi@nucleares.unam.mx
}

\begin{abstract}
Nucleon structure functions can be observed in Deep Inelastic Scattering experiments, but it is an outstanding challenge to confront them with fully non-perturbative QCD results. For this purpose we investigate the product of electromagnetic currents (with large photon momenta) between quark states (of low momenta). By means of an Operator Product Expansion the structure function can be decomposed into matrix elements of local operators, and Wilson coefficients. For consistency both have to be computed non-perturbatively. Here we present precision results for a set of Wilson coefficients. They are evaluated from propagators for numerous quark momenta on the lattice, where the use of chiral fermions suppresses undesired operator mixing. This overdetermines the Wilson coefficients, but reliable results can be extracted by means of a Singular Value Decomposition.
\end{abstract}

The XXVII International Symposium on Lattice Field Theory - LAT2009

July 26-312009

Peking University, Beijing, China

\footnotetext{
*Speaker.

${ }^{\dagger}$ Work supported by the Deutsche Forschungsgemeinschaft (DFG) through project FOR 465 "Forschergruppe Gitter-Hadronen-Phänomenologie", and through Sonderforschungsbereich SFB/TR55 "Hadron Physics from Lattice QCD”.
} 


\section{Deep Inelastic Scattering}

Historically, Deep Inelastic Scattering gave first evidence that quarks are in fact physical objects. More generally it provides insight into the hadron structure functions, and thus into the distribution of energy and spin among the hadron constituents (see Ref. [1] for a recent review).

Here we focus on the nucleon structure functions, which can be observed for instance by hard leptonic scattering dominated by one-photon exchange, as sketched below. This type of scattering only involves one quark, hence chirality is conserved.
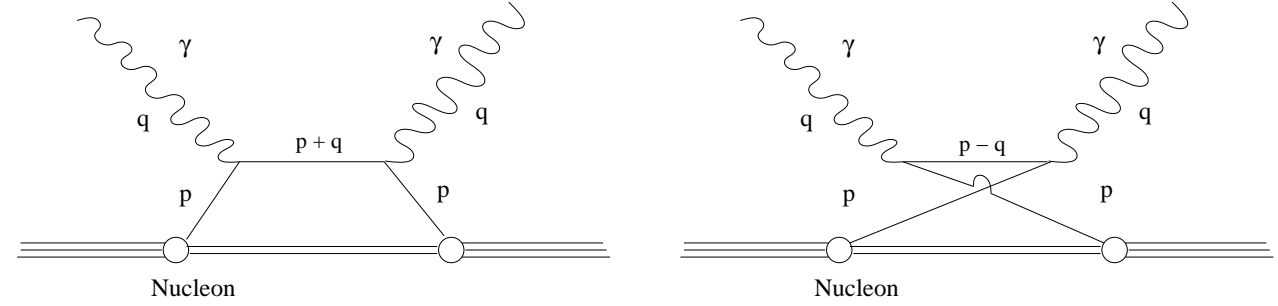

Despite the high energy in this process, perturbative QCD does not lead to fully satisfactory results. In particular, power-like IR divergences occur, which imply the notorious renormalon ambiguities [2]. A consistent evaluation of the nucleon structure function from first principles of QCD has to be fully non-perturbative. Hence it is a challenge for lattice studies, and the goal of our project.

\section{Nucleon structure functions on the lattice}

We start from the general ansatz for moments of a lattice nucleon structure function,

$$
\mathscr{M}\left(q^{2}\right)=c^{(2)}(a q) A_{2}(a)+\frac{c^{(4)}(a q)}{q^{2}} A_{4}(a)+\ldots \quad\{\text { higher twists }\}
$$

where $a$ is the lattice spacing, $q$ is the photon momentum being transferred, $c^{(n)}$ are Wilson coefficients and $A_{n}$ are matrix elements (their Lorentz structure is factored out). Traditionally the latter are computed by lattice simulations, whereas the coefficients $c^{(n)}$ are evaluated perturbatively, since they only depend on the large photon momentum. However, consistency strictly requires the nonperturbative evaluation of both factors [3]. Here we present precise numerical results which are relevant for $c^{(2)}$. Further steps in this project, leading to $\mathscr{M}\left(q^{2}\right)$, are reported in Refs. [4, 5].

\section{Lattice technicalities}

We used configurations that were generated quenched with the Lüscher-Weisz gauge action on a lattice of size $24^{3} \times 48$ at $\beta=8.45$. Based on $r_{0}=0.5 \mathrm{fm}$, the lattice spacing has been determined as $a=0.095(1) \mathrm{fm} \mathrm{[6].}{ }^{1}$ We fixed the lattice Landau gauge, which is essential for obtaining finite values for the matrix elements; moreover gauge fixing is helpful to reduce the statistical noise.

Our analysis involves two flavours of degenerate valence quarks, which are represented by overlap fermions (we apply an overlap Dirac operator constructed with a Wilson kernel and a

\footnotetext{
${ }^{1}$ The error will be ignored in the following. An alternative method based on $F_{\pi}$ yielded $a \simeq 0.105 \mathrm{fm}$ [7].
} 
negative mass shift of $\rho=1.4)$. The use of chiral quarks suppresses $O(a)$ lattice artifacts, as well as undesired operator mixing. The latter virtue is particularly important in this project; for instance it is essential to disentangle the contributions of higher twist, cf. eq. (2.1).

Our study includes two bare masses for the degenerate quark flavours, $m_{q}=0.011 \simeq 29 \mathrm{MeV} \rightarrow m_{\pi} \approx 280 \mathrm{MeV} \quad$ and $\quad m_{q}=0.028 \simeq 73 \mathrm{MeV} \rightarrow m_{\pi} \approx 440 \mathrm{MeV}$

\section{OPE on the lattice}

We use standard lattice electromagnetic currents $J_{\mu}$ (it would be computationally expensive to work with currents, which are conserved in the framework of overlap quarks). Thus one renormalisation constant $\left(Z_{V}\right)$ will be needed $[4,5]$. The Operator Product Expansion (OPE) decomposes the product of two such currents between quark states as follows,

$$
W_{\mu v} \simeq\left\langle\psi(p)\left|J_{\mu}(q) J_{v}^{\dagger}(q)\right| \psi(p)\right\rangle \overbrace{=}^{\mathrm{OPE}} \sum_{m, i, n} C_{\mu v, i, \mu_{1} \ldots \mu_{n}}^{(m)}(q)\left\langle\psi(p)\left|\mathscr{O}_{i, \mu_{1} \ldots \mu_{n}}^{(m)}\right| \psi(p)\right\rangle
$$

$C^{(m)}$ : Wilson coefficients, independent of the target and therefore of the quark momentum $p$ $\mathscr{O}^{(m)}$ : local operators, relevant to describe the nucleon structure

$\mu_{j}$ : momentum component $p_{\mu_{j}}$ appears in the operator

$i=1 \ldots 16$ : Clifford index $, m:$ index for operators with the same symmetries.

A truncation of the OPE in eq. (4.1), and small lattice artifacts, require the scale separation

$$
p^{2} \ll q^{2} \ll(\pi / a)^{2} .
$$

Assuming this separation to hold, we consider quark bilinears up to $O\left(|p|^{3}\right)$,

$$
\bar{\psi} \Gamma \psi, \quad \bar{\psi} \Gamma D_{\mu_{1}} \psi, \quad \bar{\psi} \Gamma D_{\mu_{1}} D_{\mu_{2}} \psi, \quad \bar{\psi} \Gamma D_{\mu_{1}} D_{\mu_{2}} D_{\mu_{3}} \psi .
$$

The symbol $\Gamma$ captures the full Clifford structure, hence this set includes a frightening number of $16 \cdot \sum_{d=0}^{3} 4^{d}=1360$ operators. However, we choose specific photon momenta of the diagonal form $q \propto(1,1,1,1)$, which implies a high level of symmetry. To be explicit, we consider three photon momenta,

$a q_{\mu}^{(1)}=\frac{\pi}{6},\left|q^{(1)}\right| \simeq 2.2 \mathrm{GeV}, \quad a q_{\mu}^{(2)}=\frac{\pi}{4},\left|q^{(2)}\right| \simeq 3.3 \mathrm{GeV}, \quad a q_{\mu}^{(3)}=\frac{\pi}{3},\left|q^{(3)}\right| \simeq 4.4 \mathrm{GeV}$.

For $q^{(2)}$ and $q^{(3)}$ we implement standard boundary conditions (b.c.), but $q_{\mu}^{(1)}$ is applied along with twisted b.c. for the quark fields: in addition to the Euclidean time direction, also two of the spatial directions are antiperiodic. This gives access to smaller $p$-momenta, which are needed in view of condition (4.2), since $\left|q^{(1)}\right|$ is not that large.

Thanks to our diagonal choice of $q$, the set of operators reduces to only 67 equivalence classes [8]. We classify the corresponding Wilson coefficients according to the number of derivatives in the operators that they multiply:

$C_{1}$ : no derivative, multiplies $\bar{\psi} 11 \psi$

$C_{2} \ldots C_{6}:$ one derivative, Bjorken scaling $\propto 1 / q^{2}$

$C_{7} \ldots C_{16}:$ two derivatives

$C_{17} \ldots C_{67}:$ three derivatives, Bjorken scaling $\propto 1 /\left(q^{2}\right)^{2}$.

The coefficients of operators with an even number of derivatives vanish at $m_{q}=0$ due to chiral symmetry. 
In each case, our evaluation of $C_{1} \ldots C_{67}$ involves numerous quark momenta $p_{1}, \ldots, p_{M}$, see Table 1.

\begin{tabular}{c||c|c|c|c|c|c|}
\multicolumn{1}{c||}{} & \multicolumn{2}{c|}{$q^{(1)}$} & \multicolumn{2}{c|}{$q^{(2)}$} & \multicolumn{2}{c|}{$q^{(3)}$} \\
& $M$ & $S V_{\mathrm{opt}}$ & $M$ & $S V_{\mathrm{opt}}$ & $M$ & $S V_{\mathrm{opt}}$ \\
\hline$m_{q}=0.011$ & 15 & 14 & 31 & 10 & 31 & 10 \\
\hline$m_{q}=0.028$ & 15 & 12 & 32 & 8 & 31 & 8 \\
\hline
\end{tabular}

Table 1: The number $M$ of quark momenta, and $S V_{\text {opt }}$ of Singular Values (see below) that we used for the determination of the Wilson coefficients in each case, i.e. for each quark mass and photon momentum.

For $q^{(1)}$, i.e. with twisted b.c., there are less $p$-momenta with small $p^{2}$, hence less $p$-momenta are needed for converging results. Thus we measure $W_{\mu \nu}-$ given in eq. (4.1) - off-shell for $M=$ $15 \ldots 32$ quark momentum sources to determine the Wilson coefficients $C_{1} \ldots C_{67}$. Schematically they are given as (the elements $W^{\left(p_{i}\right)}$ and $\mathscr{O}_{k}^{\left(p_{i}\right)}$ are $4 \times 4$ matrices capturing the spin components)

$$
\left(\begin{array}{c}
W^{\left(p_{1}\right)} \\
\cdot \\
\cdot \\
\cdot \\
W^{\left(p_{M}\right)}
\end{array}\right)=\left(\begin{array}{ccc}
\mathscr{O}_{1}^{\left(p_{1}\right)} & \ldots & \mathscr{O}_{67}^{\left(p_{1}\right)} \\
\cdot & \ldots & \cdot \\
\cdot & \cdots & \cdot \\
\cdot & \cdots & \cdot \\
\mathscr{O}_{1}^{\left(p_{M}\right)} & \ldots & \mathscr{O}_{67}^{\left(p_{M}\right)}
\end{array}\right)\left(\begin{array}{c}
C_{1} \\
\cdot \\
\cdot \\
C_{67}
\end{array}\right)
$$

Since $16 M \gg 67$ in all our cases, the system is strongly over-determined. Hence we apply a Singular Value Decomposition: it selects the $n \leq 67$ conditions with "maximal impact" on the solution $C_{1} \ldots C_{67}$. We order the corresponding Singular Values (SV, analogues to eigenvalues) hierarchically. If their magnitude drops rapidly one has favourable conditions to extract a reliable result. Fig. 1 illustrates that this is in fact the behaviour that we observed.
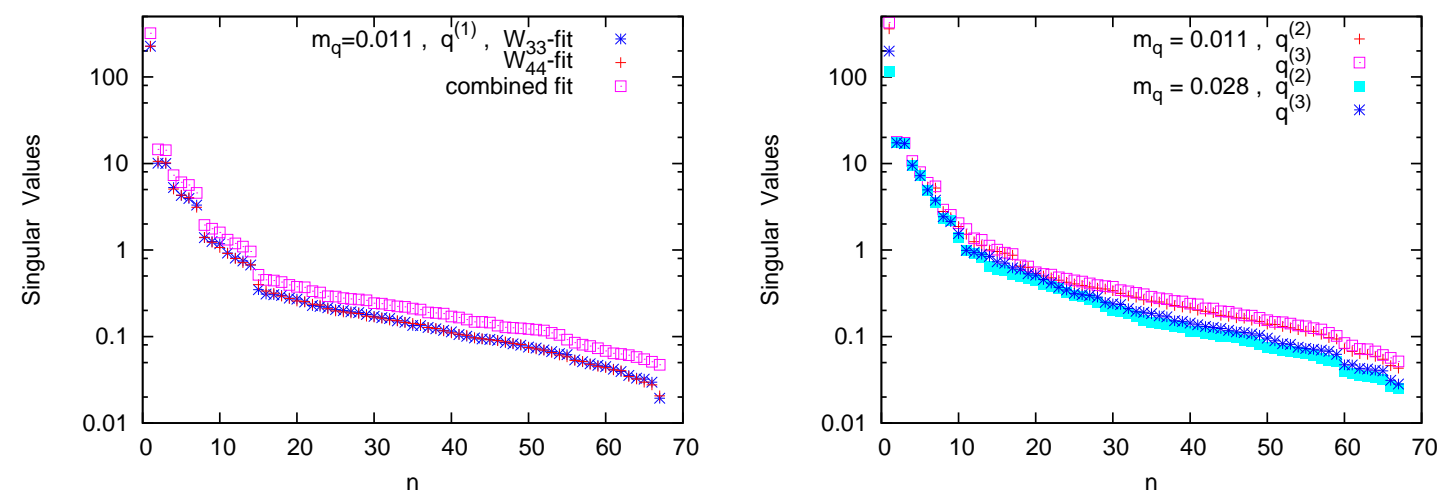

Figure 1: Examples for the rapidly dropping magnitudes of the SV. Due to reflection and rotation symmetries some Wilson coefficients coincide theoretically, e.g. the coefficients of $\mathscr{O}_{i, 33}$ in $W_{33}$, and of $\mathscr{O}_{i, 44}$ in $W_{44}$. We determine the corresponding SV separately, and by a combined fit, which implements this identity. Left: separate and combined SV for $m_{q}=0.011, q^{(1)}$. Right: combined SV for both quark masses and $q^{(2)}, q^{(3)}$.

As our next criterion, Fig. 2 shows how the squared residues in eq. (4.4) decrease as the number of SV involved rises from $n=1 \ldots 67$.

\section{Results for the Wilson coefficients}

Solving eq. (4.4) for $C_{1} \ldots C_{67}$ employs the inverse SV, so including all of them is not optimal: the tiny SV, with large relative noise, tend to distort the result. Therefore we computed the Wilson 

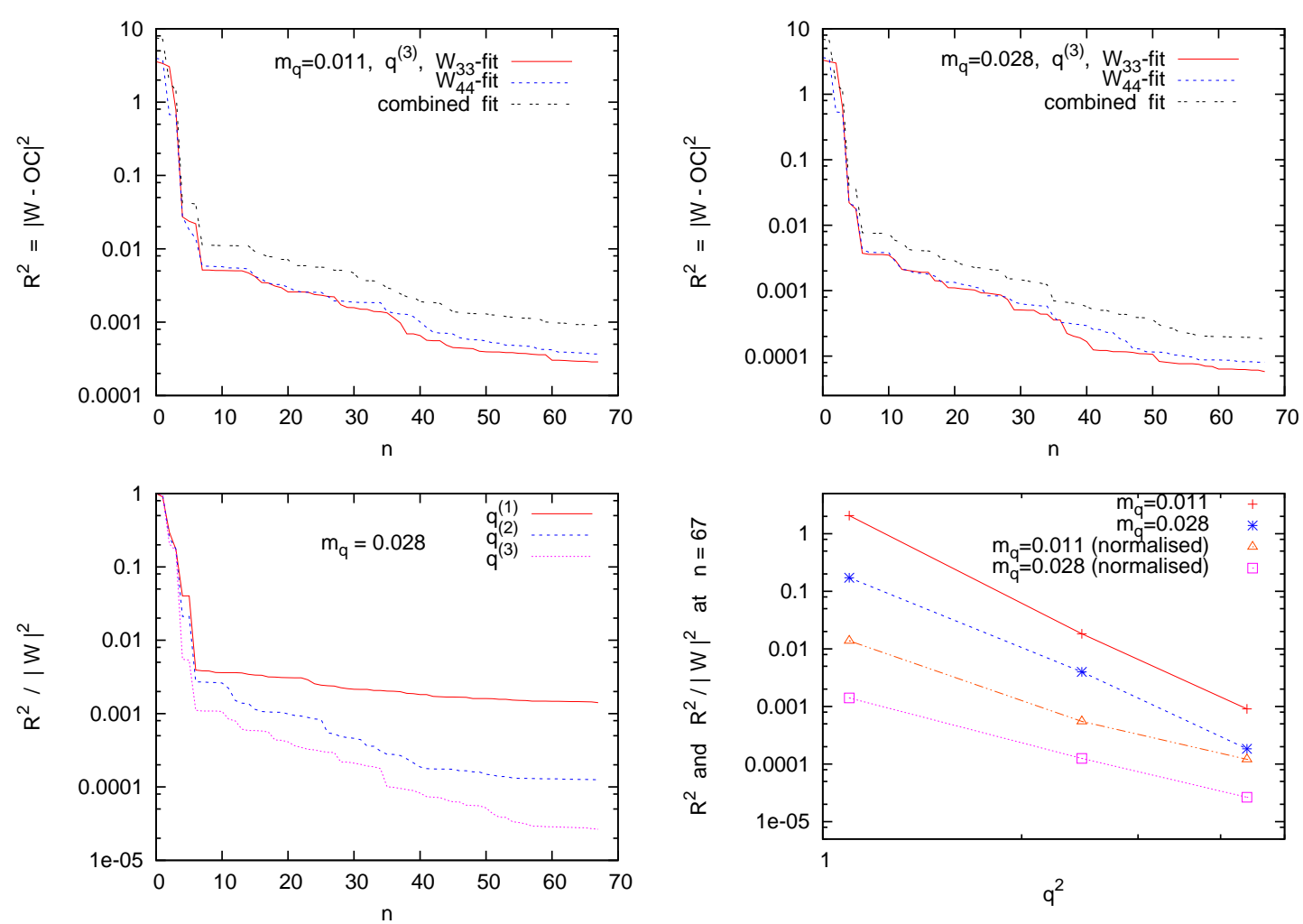

Figure 2: Squared residues $R^{2}$ for the operator $\vec{\psi} \vec{\gamma} \vec{D} \psi$ (as an example) against the number $n$ of conditions (corresponding to the SV). The plots above show results of separate and combined fits (as in Fig. 1), for $q^{(3)}$ and $m_{q}=0.011$ (left), and $m_{q}=0.028$ (right). The plot below on the left shows the normalised $R^{2}$ for combined fits at $m=0.028$. The final plateau value (at $n=67$ ) is compared in the plot below on the right: its decrease for increasing $q^{2}$ is roughly linear, so that the relative error remains approximately constant.

coefficients with a gradually increasing number of SV, $n=1 \ldots 67$; an example is shown in Fig. 3 (left). It displays the most important coefficients, i.e. those of operators with one derivative. The only common plateau occurs in the range of $7 \ldots 13 \mathrm{SV}$ included. To check if this plateau holds for all 67 coefficients, we compare the full set obtained with 7, 10 and 13 SV in Fig. 3 (right). We observe a striking confirmation of this plateau. The results look similar for other $m_{q}$ and $q$. The optimal number of SV in each case, considering also the impact on $\mathscr{M}$ [5], is displayed in Table 1.

Next we verify if our number of quark momenta is sufficient: we check if the results change significantly as we omit part of them. Fig. 4 shows (with examples) that this is not the case here: convergence for an increasing number of $p$-momenta is well confirmed.

In Fig. 5 (plots above) we compare our results for the Wilson coefficients at $q^{(2)}$ to the corresponding values at tree level. As we mentioned in Section 4, $C_{1}$ (which multiplies $\bar{\psi} 11 \psi$ ) and $C_{7} \ldots C_{16}$ (attached to operators $\mathscr{O}^{(m)}$ with $\gamma_{\mu} \gamma_{v}$ ) vanish at tree level in the chiral limit. We measured consistently small values for these coefficients, which indicates that approximate chirality and operator mixing are indeed under control, in contrast to previous studies with Wilson fermions [9]. Generally the measured Wilson coefficients follow the same pattern as their counterparts on tree level, though with significantly reduced absolute values.

Fig. 5 (plots below) show the commonly applied Wilson coefficients ratios $C_{m} / C_{m}^{\text {tree level }}$. The dependence on the quark mass is weak, which approves again a safe approximate chirality. On the 

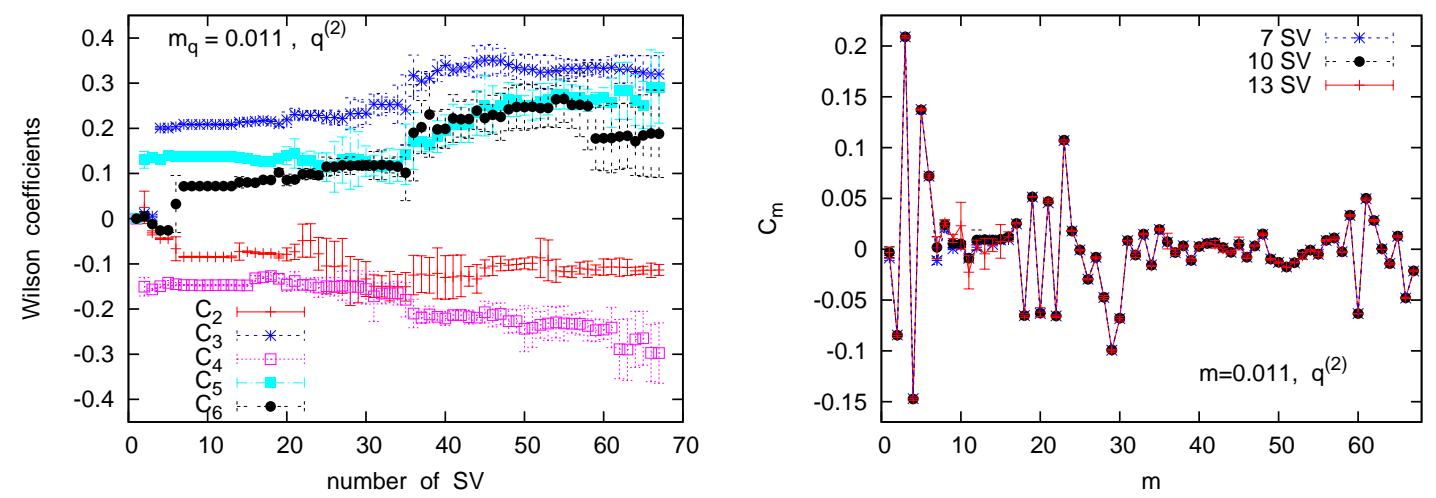

Figure 3: Results for the Wilson coefficients at $m_{q}=0.011$ and $q^{(2)}$ as a varying number of SV is included. Left: the coefficients to the 1-derivative operators for $n=1 \ldots 67$ reveal a single plateau for $n=7 \ldots 13$. Right: the full set $C_{1} \ldots C_{67}$ agrees very well for $n=7,10$ and 13 , confirming this plateau generally.
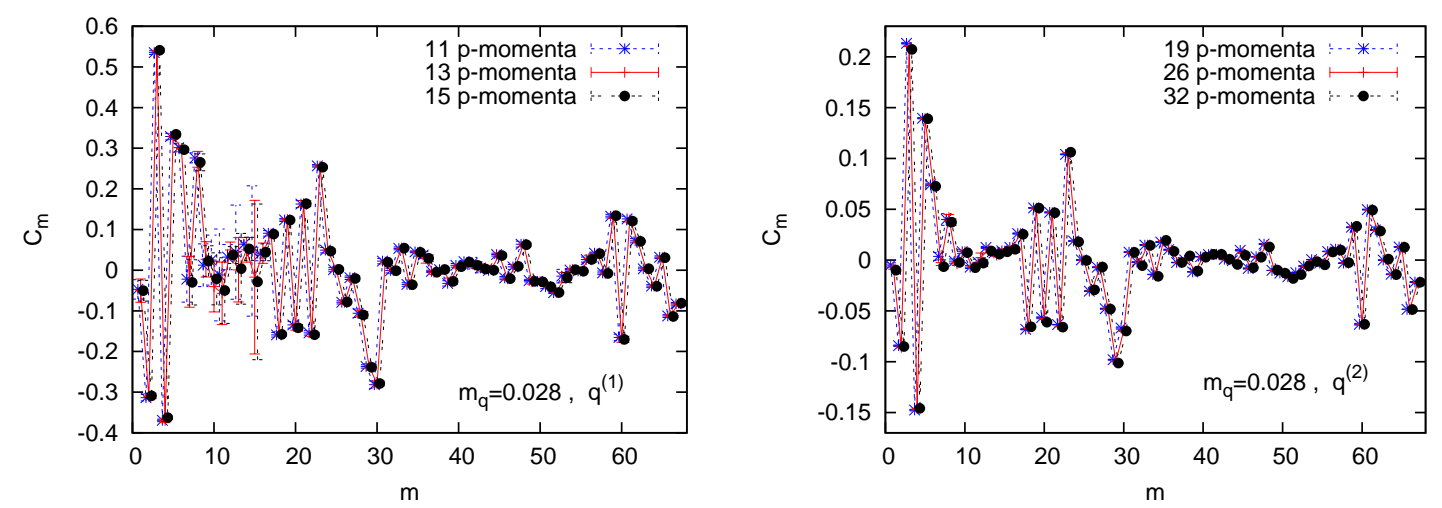

Figure 4: The Wilson coefficients determined from a varying number of the quark momenta. We show results for $m_{q}=0.028$, at $q^{(1)}$ with 11,13 or $15 p$-momenta (left) and at $q^{(2)}$ with 19,26 or $32 p$-momenta (right). This hardly changes the results for the Wilson coefficients, hence their convergence appears safe.

other hand, we observe a strong dependence on the photon momentum $q$, as expected. Ref. [5] discusses the detailed comparison with the theoretically expected Bjorken scaling behaviour (cf. Section 4).

\section{Conclusions and outlook}

We have evaluated a set of Wilson coefficients non-perturbatively (partial result were anticipated in Refs. [8]). They refer to twist 2; for the photon momenta that we used, contributions by higher twists are suppressed [9]. The application of chiral lattice quarks has been crucial to control the operator mixing. We demonstrated in detail that our results are reliable regarding the number of SV and quark momenta included in the evaluation. The measured Wilson coefficients follow the pattern of their counterparts at tree level, though with smaller absolute values.

The structure function $\mathscr{M}$ (in eq. (2.1)) is now obtained by means of Nachtmann integration over $W_{\mu v}$ (cf. eq. (4.1)). This is worked out for a single quark in Ref. [4]. The final step to a fully non-perturbative moment of the nucleon structure function — given by products between the matrix elements [10] and the Wilson coefficients presented here — is carried out in Refs. [5, 11]. 

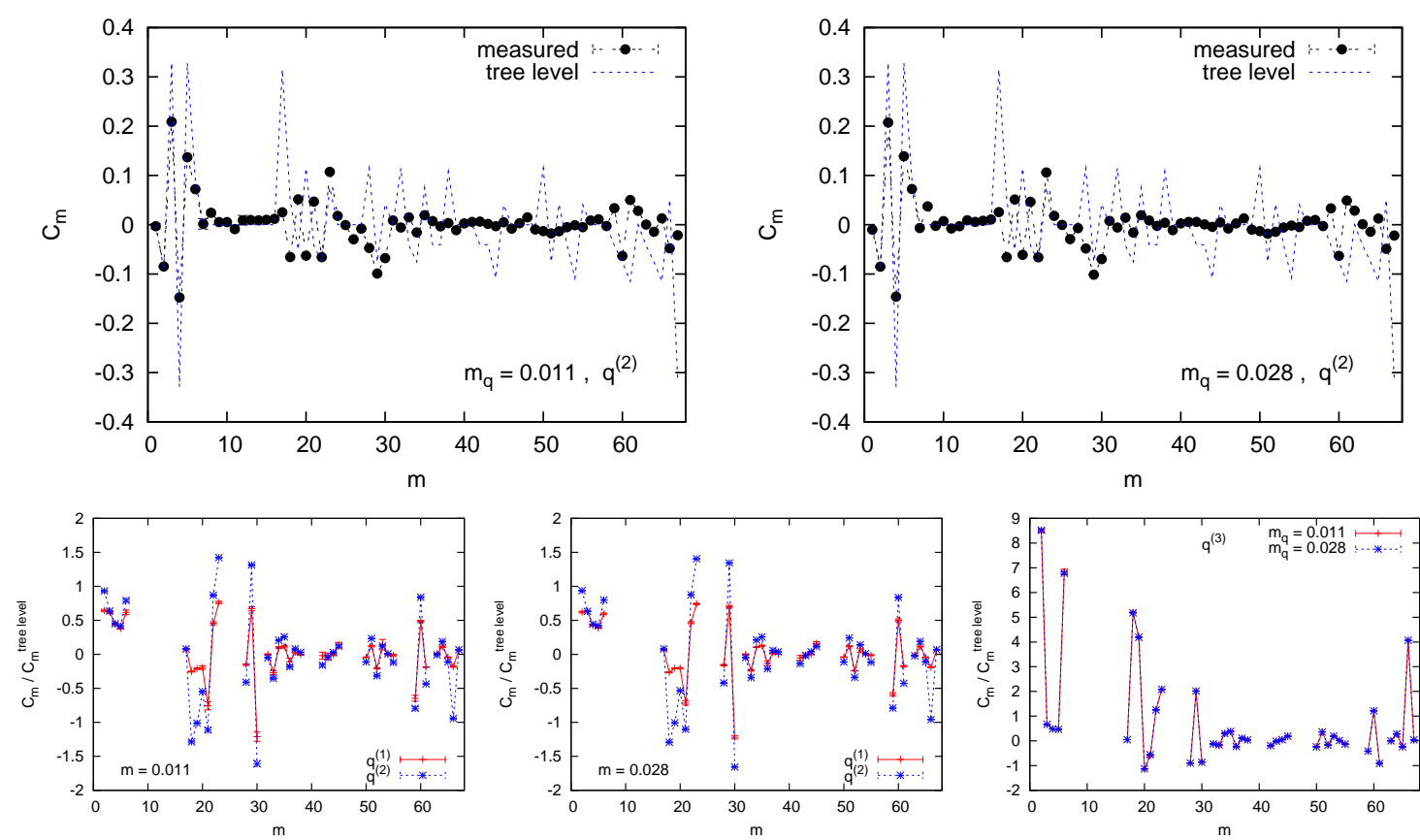

Figure 5: Above: measured Wilson coefficients compared to their tree level values for $q^{(2)}$. Coefficients for operators with an even number of derivatives take consistently small values. Below: the ratios $C_{m} / C_{m}^{\text {tree level }}$ (for non-vanishing denominators). They depend only mildly on the quark mass.

Acknowledgement : The computations for this project were performed on the clusters of the "Norddeutscher Verbund für Hoch- und Höchstleistungsrechnen" (HLRN).

\section{References}

[1] C. Diaconu, Int. J. Mod. Phys. A 24 (2009) 1069.

[2] A.I. Vainshtein and V.I. Zakharov, Phys. Rev. Lett. 73 (1994) 1207. (erratum ibid. 75 (1995) 3588). E. Stein, M. Maul, L. Mankiewicz and A. Schäfer, Nucl. Phys. B 536 (1998) 318. V.M. Braun, E. Gardi and S. Gottwald, Nucl. Phys. B 685 (2004) 171.

[3] G. Martinelli and C.T. Sachrajda, Nucl. Phys. B 478 (1996) 660.

[4] Talk presented by P.E.L. Rakow at LATTICE09, write-up in these proceedings.

[5] Talk presented by T. Streuer at LATTICE09, write-up in these proceedings.

[6] C. Gattringer, R. Hoffmann and S. Schaefer, Phys. Rev. D 65 (2002) 094503.

[7] M. Gürtler, R. Horsley, P.E.L. Rakow, C.J. Roberts, G. Schierholz and T. Streuer, PoS(LAT2005)125.

[8] W. Bietenholz, N. Cundy, M. Göckeler, R. Horsley, H. Perlt, D. Pleiter, P.E.L. Rakow, C.J. Roberts, G. Schierholz, A. Schiller and J.M. Zanotti, PoS(LAT2007)159. W. Bietenholz, N. Cundy, M.

Göckeler, R. Horsley, H. Perlt, D. Pleiter, P.E.L. Rakow, G. Schierholz, A. Schiller and J.M. Zanotti, PoS(LAT2008)149.

[9] S. Capitani, M. Göckeler, R. Horsley, H. Oelrich, D. Petters, P.E.L. Rakow and G. Schierholz, Nucl. Phys. (Proc. Suppl.) 73 (1999) 288. D. Petters, Ph.D. thesis, Freie Universität Berlin (2000).

[10] D. Galletly, M. Gürtler, R. Horsley, K. Koller, V. Linke, P.E.L. Rakow, C.J. Roberts, G. Schierholz and T. Streuer, PoS(LAT2005)363.

[11] QCDSF Collaboration, in preparation. 